\title{
Multimodal identity tracking in a smart room
}

\section{Keni Bernardin · Hazim Kemal Ekenel •}

Rainer Stiefelhagen

Published online: 8 January 2009

(C) Springer-Verlag London Limited 2008

Erratum to: Pers Ubiquit Comput (2009) 13:25-31

DOI 10.1007/s00779-007-0175-y

Due to a processing error, the spelling of one author name was incorrect in this article. The correct name should read Rainer Stiefelhagen.

The online version of the original article can be found under doi:10.1007/s00779-007-0175-y.

K. Bernardin $(\bowtie) \cdot$ H. K. Ekenel · R. Stiefelhagen Universitaet Karlsruhe, ITI, 76131 Karlsruhe, Germany

e-mail: keni@ira.uka.de

H. K. Ekenel

e-mail: ekenel@ira.uka.de

R. Stiefelhagen

e-mail: stiefel@ira.uka.de 